\title{
Hair follicle associated neural crest stem cells interact with mouse brain tissue
}

\author{
Arkadii Beilin ${ }^{1}$, Anastasiia Kosykh ${ }^{1}$, Kirill Sukhinich ${ }^{1}$, Ekaterina Vorotelyak ${ }^{1}$ \\ 1. Laboratory of Cell Biology, Koltzov Institute of Developmental Biology of \\ Russian Academy of Sciences, Moscow, Russian Federation.
}

\section{Introduction}

Hair follicle contains population of EPI-NCSCs (Epidermal Neural Crest Stem Cells) which can be isolated in culture and differentiated in neural direction. EPI-NCSCs can provide new available source of stem cells for autologous therapy of neurodegenerative diseases.

In this work we show how EPI-NCSCs interact with brain tissue.

\section{Material and Methods}

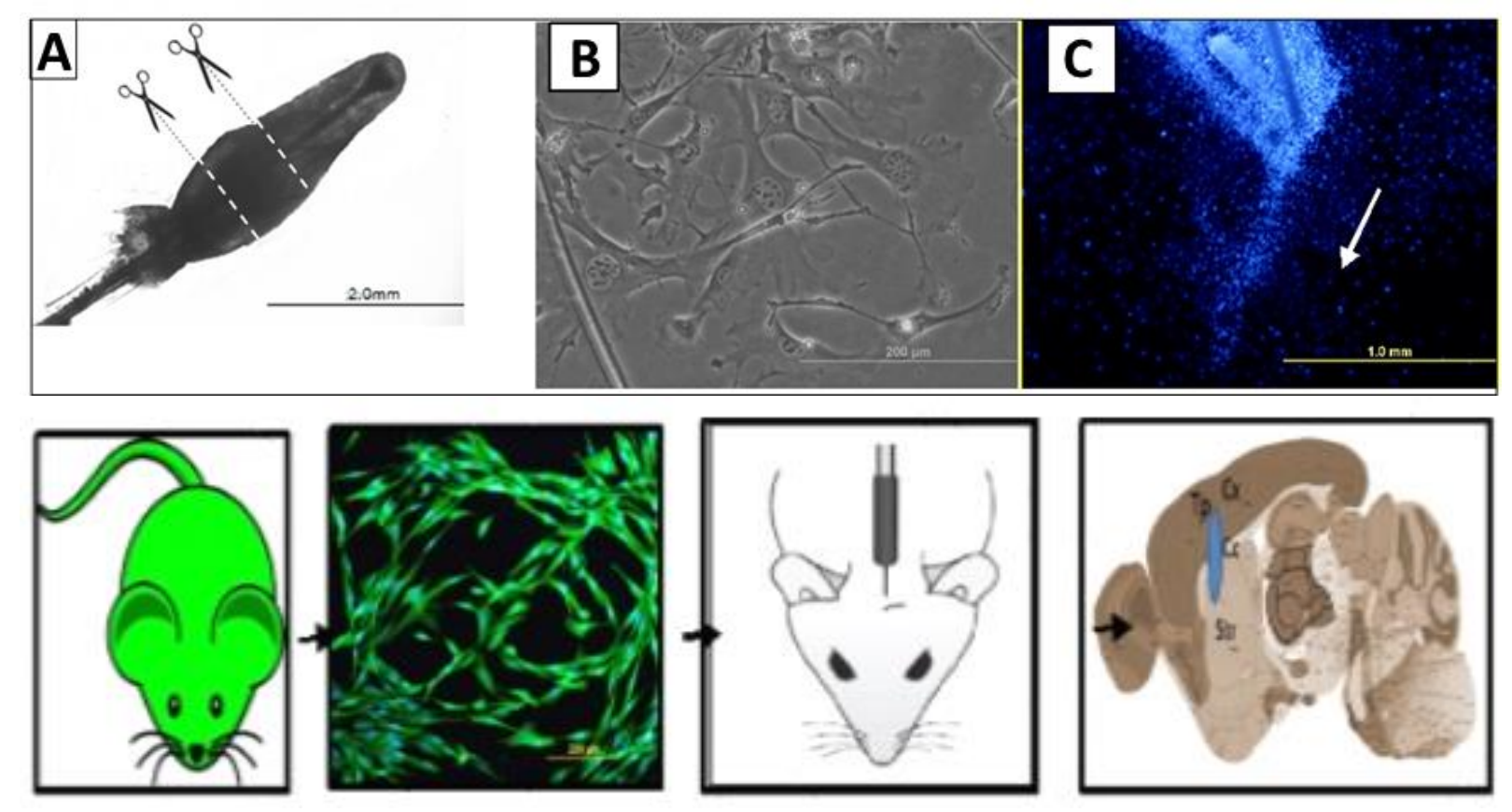

Results

Primary EPI-NCSCs cultures expressed NCSCs markers (nestin, neurotrophin receptor $\mathrm{p} 75$, sox10), neuronal and glial - specific markers (beta III tubulin, myelin basic protein), and stem cell marker - fibronectin.

Migration (upper fig.) and scattering (lower fig.) analyses showed that conditioned by embryonic tissue medium accelerated migration of EPI-NCSCs in the first two days, but then migration capacity significantly decreased in age depended manner.
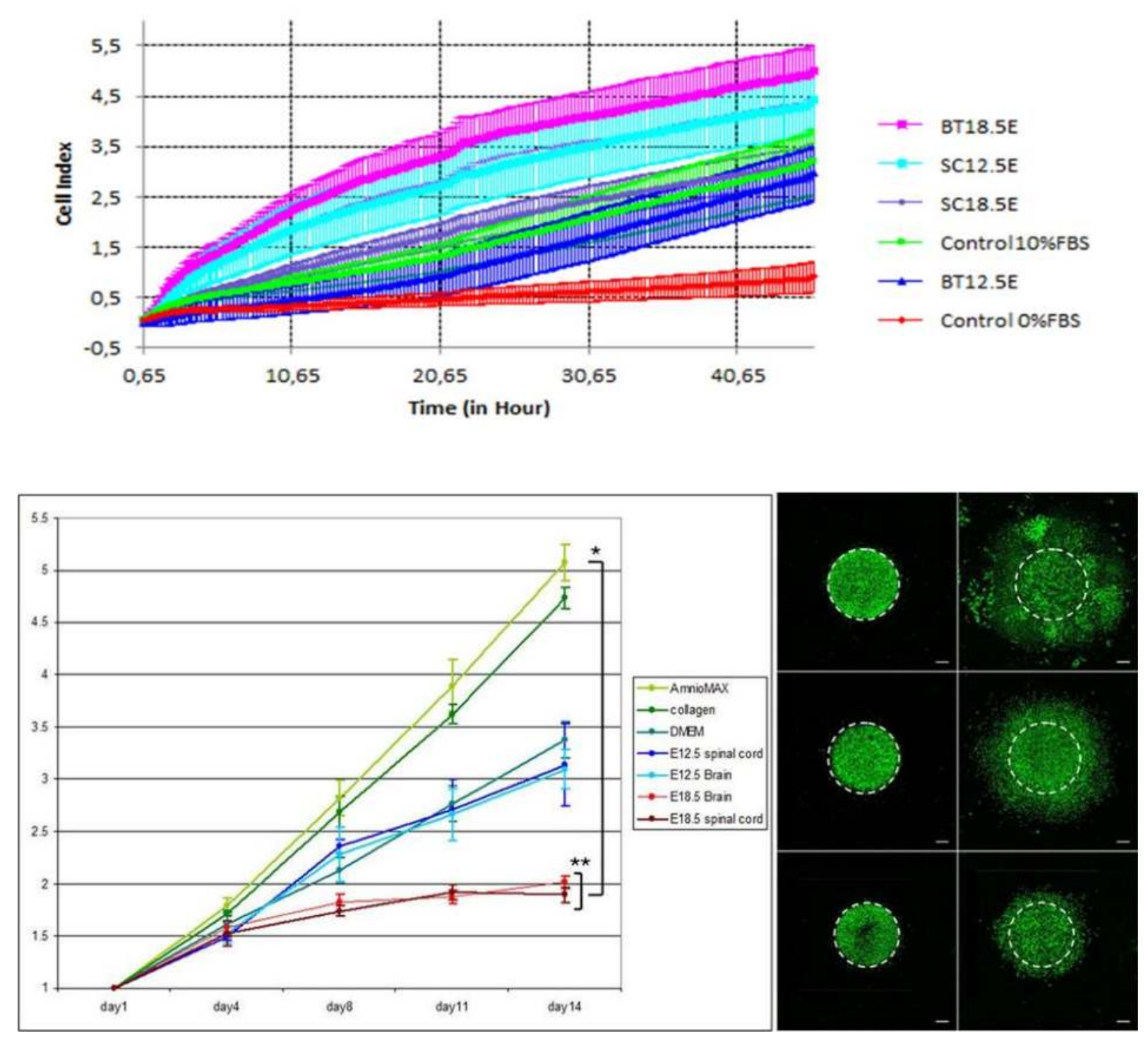

Research was funded by program of fundamental research of RAS presidium "Fundamental research for biomedical technologies development"

\section{Results}

Analysis of ki67 expression showed that EPI-NCSCs proliferation activity diminishes in culture, but SC18.5E provides the rise of EPI-NCSCs proliferation over time.

Cultivation EPI-NCSCs on thick brain slices showed that during first 4 days primary HF-NCSCs proliferated actively, and to day 7 the proliferation level significantly decreased by more than 3 times. Also doublecortin expression was observed in some EPINCSCs.

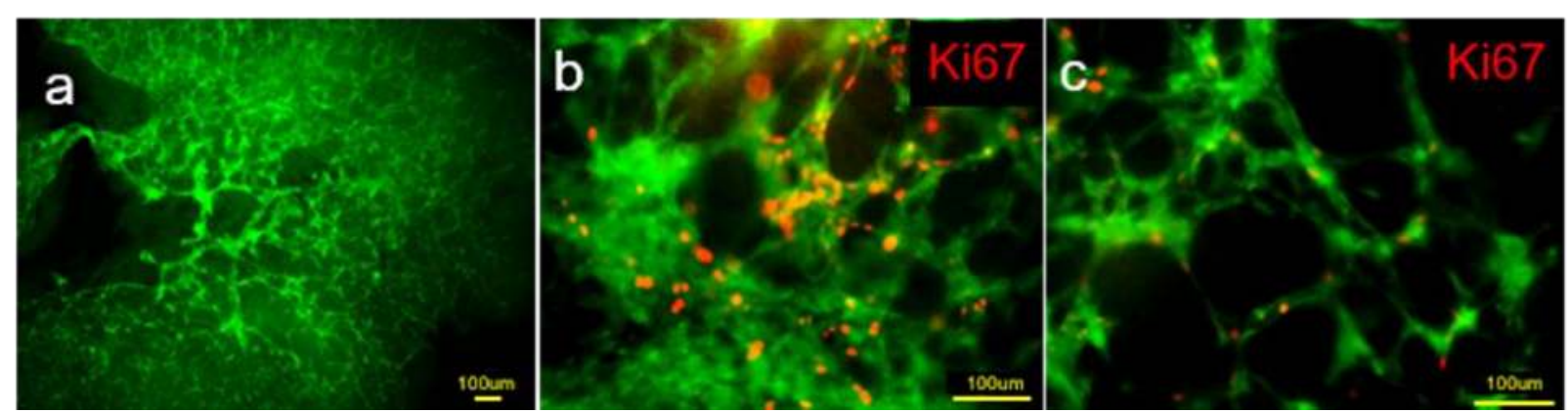

Differentiated into neural direction EPI-NCSCs started to express NeuN (Neuron Nuclei) and doublecortin.

Transplanted into adult mouse brain EPI-NCSCs were alive at $21^{\text {st }}$ day after transplantation, while differentiated EPI-NCSCs were alive at $28^{\text {th }}$ day after transplantation.
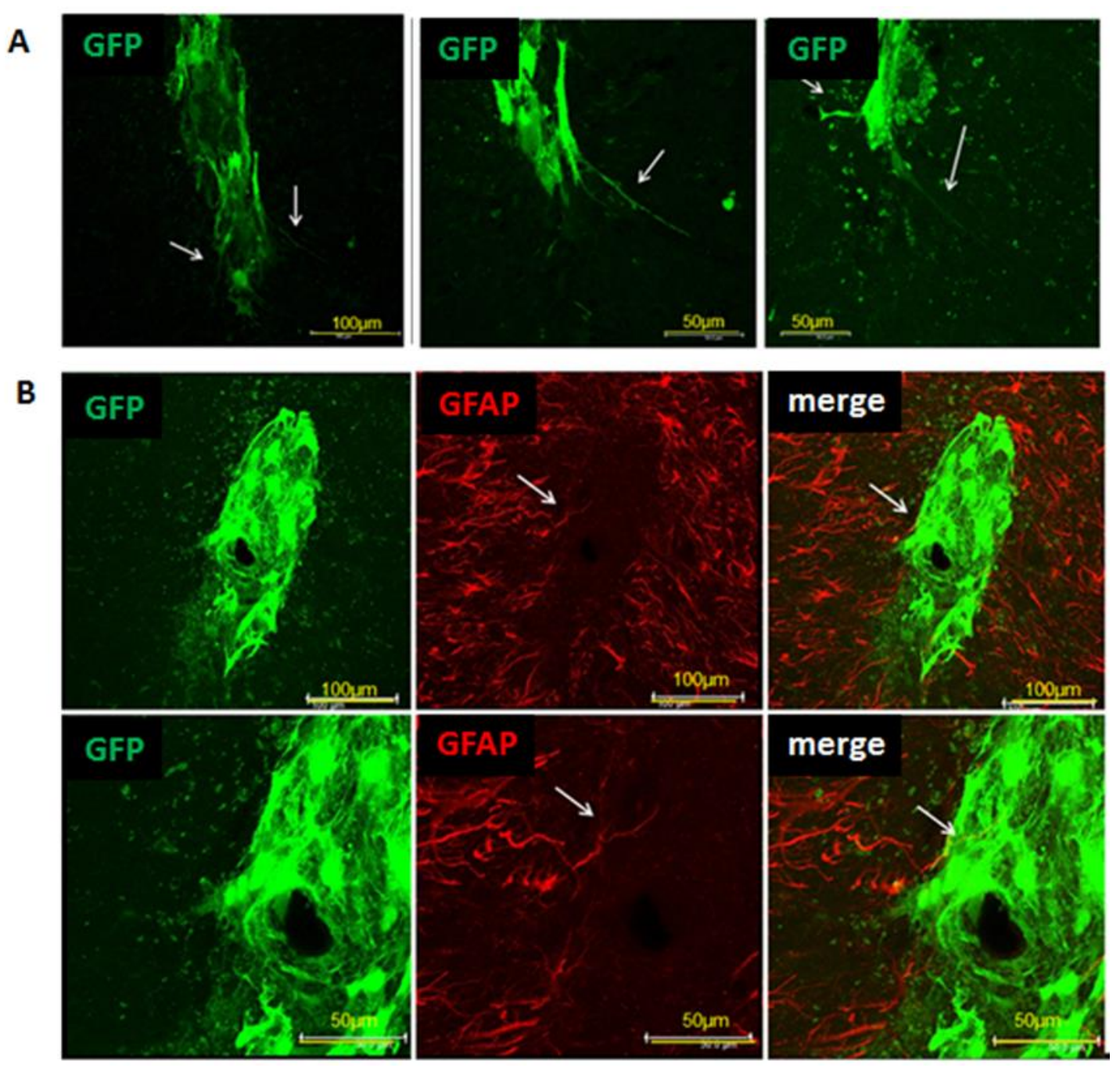

\section{Conclusions}

Migration capacity of the HF-NCSCs corresponds to the stage but not type of neural tissue development present in co-culture, on the early stages migration is higher than on the later one.

In contrast, interaction with adult brain changes HF-NCSCs status and reduces proliferation.

Differentiated HF-NCSCs grafts survive longer in brain transplants and have higher opportunity for migration than primary ones. 